\title{
A Western Blot and Immunoprecipitation Assay to Verify Antibody Specificity
}

Sharon Bonnette, Ph.D., Bethyl Laboratories, Inc.

\section{Abstract}

Life scientists are raising awareness of the problem of irreproducible data in basic research (1) (2). Part of the crisis of irreproducibility has been blamed on antibodies (3), and from this standpoint, an equal push to bring awareness to antibody validation has been initiated (4) (5) (6) (7). Although scientists agree the onus of validation is on the user, it is also viewed that commercial antibody providers should share in this responsibility (4) (6) (8) (7). For companies who try to provide breadth in their catalog, validation is not inconsequential. It presents a difficult and costly impediment; nevertheless, users deserve transparency and a genuine effort from vendors to qualify their products. The first and most important quality of validation is verifying the antibody recognizes the intended target. The most accepted methods for validating antibody specificity include application-specific testing using knockout $(\mathrm{KO})$ or RNA knockdown samples and/or using multiple antibodies against distinct epitopes of the intended target (6) (9). This paper describes Bethyl Laboratories' solution for a practical, high-throughput method for validating the target-specificity of antibodies for the application of western blot (WB). The method involves verification of specificity via WB of immunoprecipitates using two or more antibodies that recognize distinct epitopes of a target protein.

\section{Introduction - The Challenge}

Verifiable and reproducible data is essential to support downstream discovery in the prevention, diagnosis, and treatment of disease. When scientists cannot verify or reproduce basic and pre-clinical data, research dollars are squandered and discovery is delayed (1). Poorly characterized antibody reagents have been implicated as a source of scientific irreproducibility. This is evident in the increasing presence of editorials and sponsored webinars on the topic (10) (11) and changes in requirements by journals to require authors to validate and properly describe their reagents (12) (6). Researchers are highly dependent on commercial antibodies-about 95\% of antibody users rely on premade antibodies (13). Regrettably, life scientists have little trust in the quality of these antibodies (14) (15) (9), and validation by these providers is described as "usually inadequate and frequently unreliable" (6). Owing to this perception, the effort and financial investment required by users for procuring commercial antibodies can be a frustrating process, especially when a reagent is not well-described in the literature.

\section{The Solution - Meeting the Challenge}

Surprisingly, although there are a large number of commercial antibody sources, there is still a desperate need for affinity binding reagents (16). The need stems from two problems: varying quality and lack of proteome coverage. For many manufacturers, generating antibodies is not the rate-limiting step for obtaining a large catalog of useful products. The major constraint is proving they recognize the intended target and defining the utility of the antibodies. In order to make qualified reagents that accelerate discovery in basic or pre-clinical research, high-throughput manufacturing followed by high-throughput validation is necessary. To achieve this, Bethyl Laboratories uses a practical and efficient test to correctly identify the intended target band developed in WB assays and verify antibody specificity. It is based on the theory that an antibody will recognize the immunoprecipitate of an antibody raised against an alternative epitope. The test involves WB and immunoprecipitation (IP) assays using multiple epitope-specific antibodies directed at the same target protein.

The process begins with designing and synthesizing two to four distinct peptide antigens. Antibodies against the distinct antigens are then raised in rabbits and antigen-affinity purified (Fig. 1A). To verify specificity, IP and WB assays are conducted. Each antibody is used in separate reactions to immunoprecipitate endogenously expressed target protein from a positive lysate. The group of distinct Immunoprecipitates is then separated on SDS-PAGE gels and transferred. Replicate blots are made and each blot is individually incubated with an epitope-specific antibody (Fig 1B). The band recognized by an antibody raised against a distant epitope is identified as the intended target band. (Fig. 1B). Once the target band is correctly identified, the antibodies are tested further to assess their performance and specificity in WB of whole cell lysates (Fig 1C). For final qualification, the antibody must recognize the specific target band (as identified in the immunoprecipitate) and exhibit minimal non-confounding cross-reactivity. 


\section{Conclusion}

Antibody validation can be challenging but is requisite for the generation of reliable data. Users and providers share in the responsibility of verifying the specificity of antibodies. It can be difficult to test for specificity using knockout tissue or RNA knockdown lysate. Using multiple antibodies to distinct epitopes in a combined WB/IP assay is an effective alternate method for verifying specificity. This method allows Bethyl Laboratories to support and accelerate discovery by efficiently providing qualified antibody reagents for basic and pre-clinical research.

Bethyl Laboratories, Inc. offers over 5,200 trial-sized antibodies to more than 2,700 protein targets. Since its founding in 1972, Bethyl has manufactured all of its antibodies on-site in Texas to exacting standards with validation to a range of applications. Conveniently priced trial-sizes are available. To learn more, please visit: bethyl.com/ trialsize.

\section{References}

1. The Economics of Reproducibility in Preclinical Research. Freedman, Leonard P, Cockburn, lain M and Simcoe, Timothy S. 6, 2015, PLoS Biology, Vol. 13, p. e1002165.

2. A Survey on Data Reproducibility in Cancer Research Provides Insights into Our Limited Ability to Translate Findings from the Laboratory to the Clinic. Mobley, Aaron, et al. 5, 2013, PLoS, Vol. 8, p. e63221.

3. Reproducibility crisis: Blame it on the antibodies. Baker, Monya. May 21, 2015, Nature, Vol. 521, pp. 274-276.

4. Antibody validation. Bordeaux, Jennifer, et al. 3, March 2010, BioTechniques, Vol. 48, pp. 197-209.

5. Antibodies as Valuable Neuroscience Research Tools versus Reagents of Mass Distraction. Rhodes, Kenneth J and Trimmer, James S. 31, s.l. : The Journal of Neuroscience, August 2, 2006, Vol. 26, pp. 8017-8020.

6. Editorial: Antibody Can Get It Right: Confronting Problems of Antibody Specificity and Irreproducibility. Schonbrunn, Agnes. 9, s.l. : Molecular Endocrinology, 2014, Vol. 28, pp. 1403-1407.

7. Working towards a consensus for antibody validation. Reiss, Peter D, Min, Danxi and Leung, Mei Y. 266, s.l. : F1000Research, Vol. 3.

8. Commercial Antibodies: The Good, Bad, and Really Ugly. Couchman, John R. 1, s.l. : J Histochem Cytochem, 2009, Vol. 27, pp. 7-8.

9. How reliable are G-protein-coupled receptor antibodies? Michel, Martin C, Wieland,

Thomas and Gozoh, Tsujjmoto. 4, s.l. : Naunyn-Schmied Arch Pharmacol, 2009, Vol. 379, pp. 385-388.

10. Begley, Glenn C, Rimm, David L and Bandrowski, Anita. The Scientist Webinar: Validating Antibodies: An Urgent Need. s.I. : The Scientist, December 1, 2014.

11. McNutt, Marcia, et al. Science Webinar Series: Antibody validation roundtable specificity + sensitivity + reproducibility = validation. s.l. : Science, 2014 . Vol. 346.

12. An open letter to our readers on the use of antibodies. Saper, CB. 4, 2005, J Comp Neurol., Vol. 493, pp. 477-478.

13. Biolnformatics, LLC. The 2012 Market for Antibodies. Arlington : Bioinformatics, 2012.

14. Commercial antibodies and their validation [v2; ref status: indexed, http://f1000r.es/4jp]. JLA, Voskuil. 232, s.I. : F1000Reasearch, 2014, Vol. 3.

15. Reproducibility: Standardize antibodies used in research. Bradbury, Andrew and Pluckthun, Andreas. s.l. : Nature, February 5, 2015 , Vol. 518, pp. 27-29.

16. Affinity reagent resources for human proteome detection: Initiatives and perspectives. Stoevesandt, Oda and Taussig, Michael J. 2007, Proteomics, Vol. 7, pp. 2738-2750

BioTechniques 59:168-169 (September 2015) doi 10.2144/000114332
A.

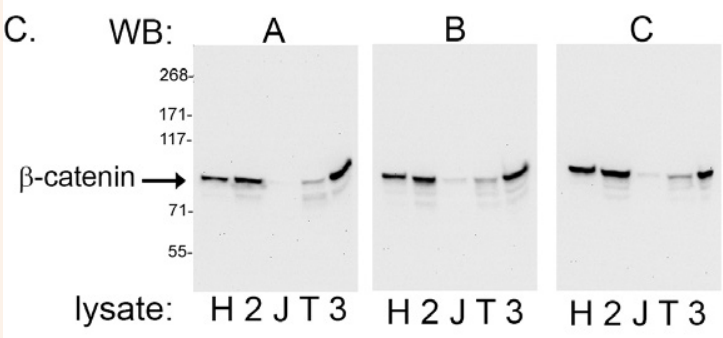

Figure 1. A western blot/immunoprecipitation assay verifies antibody specificity. A. Three distinct peptides representing epitopes of beta-catenin were synthesized as antigens for polyclonal antibody polyclonal antibodies (pAb) A302-010A, A302-011A, and A302$012 A$ respectively. $B$. Each antibody was used to immunoprecipitate endogenous beta-catenin from HeLa whole cell lysate. The immunoprecipitates were loaded as samples in western blots $(i=$ lysate; $A=I P$ with pAb against epitope $A ; B=I P$ with $p A b$ gainst epitope $B ; C=I P$ with $p A b$ antibody against epitope $C ; g=$ , Each blot was incubated with $\mathrm{pAb}$ against , $C$. All three antibodies were able to recognize beta-catenin, used to western blot whole cell lysates $(\mathrm{H}=\mathrm{HeL}$; $2=293 \mathrm{~T}$; Jurkat; T = TCMK; $3=3$ T3). All three antibodies recognized similar patterns of expression in the various lysates. 